\title{
Thermodynamics of the insulator-metal transition in dense liquid deuterium
}

\author{
M. P. Desjarlais ๑* and M. D. Knudson $\odot$ \\ Sandia National Laboratories, Albuquerque, New Mexico 87185, USA \\ R. Redmer $\odot$ \\ Institut für Physik, Universität Rostock, Albert-Einstein-Strasse 23, D18059 Rostock, Germany
}

(Received 18 December 2019; revised manuscript received 26 February 2020; accepted 27 February 2020; published 16 March 2020)

\begin{abstract}
Recent dynamic compression experiments [M. D. Knudson et al., Science 348, 1455 (2015); P. M. Celliers et al., Science 361, 677 (2018)] have observed the insulator-metal transition in dense liquid deuterium, but with an approximately 95-GPa difference in the quoted pressures for the transition at comparable estimated temperatures. It was claimed in the latter of these two papers that a very large latent heat effect on the temperature was overlooked in the first, requiring correction of those temperatures downward by a factor of 2 , thereby putting both experiments on the same theoretical phase boundary and reconciling the pressure discrepancy. We have performed extensive path-integral molecular dynamics calculations with density functional theory to directly calculate the isentropic temperature drop due to latent heat in the insulator-metal transition for dense liquid deuterium and show that this large temperature drop is not consistent with the underlying thermodynamics.
\end{abstract}

DOI: 10.1103/PhysRevB.101.104101

\section{INTRODUCTION}

The long-standing quest to produce metallic hydrogen in the laboratory has progressed rapidly in the past few years. Recent improvements in static compression techniques, using diamond anvil cells coupled with pulsed laser heating, have enabled investigation of the insulator-metal transition both in the cold, dense fluid [1-5] and in the solid [6]. Of the various attempts to achieve metallization in hydrogen, two experimental approaches have used dynamic compression of liquid deuterium to produce multiple-megabar pressures $[7,8]$. Similar in concept, these experiments were designed to probe the metallization of dense liquid deuterium by inducing a small shock followed by nominally isentropic compression. Through this combination of shock and ramp compression, the experiments, by design, avoid the solid phases of deuterium entirely while still maintaining a compression path well below the theoretical critical point temperature on the first-order insulator-metal phase transition boundary.

Experiments on the Sandia $\mathrm{Z}$ machine, as reported in Knudson et al. [7], observed indications of a first-order insulator-metal transition in dense liquid deuterium at around 280 to $305 \mathrm{GPa}$. Subsequent dynamic compression experiments at the National Ignition Facility (NIF), reported in Celliers et al. [8], also found indications of an insulator-metal

*mpdesja@sandia.gov

Published by the American Physical Society under the terms of the Creative Commons Attribution 4.0 International license. Further distribution of this work must maintain attribution to the author(s) and the published article's title, journal citation, and DOI. transition, but with a reported pressure of around $200 \mathrm{GPa}$. In the $\mathrm{Z}$ experiments, the pressure of the transition was deemed to be marked by the rapid drop in reflectivity upon release from a saturated high-reflectivity state; it was argued that this provided the clearest signature of the transition, as transients in the system (particularly due to the effects of the thermal condition) would have ample time to damp out during the several tens of nanoseconds during which the system was in the metallic state. Conversely, in the NIF experiments the transition was denoted to correspond to a reflectivity value of $30 \%$ during the initial rise in reflectivity of the compressed deuterium and did not provide data on the release from high pressure. In this context, it is worth noting that the $\mathrm{Z}$ experiments indicate both a rise in reflectivity with increasing pressure and the abrupt fall with decreasing pressure. In principle, one can argue that the $\mathrm{Z}$ experiments also express the full extent of the phase boundary on the return back to low reflectivity.

Direct measurements of the temperature were not made in either of these experiments. Instead, the temperature was estimated using an equation of state (EOS) model for the shock ring up, followed by estimates of the temperature increase for the quasi-isentropic ramp compression path using either (i) first-principles calculations (Z experiments) or (ii) an average of three tabulated EOS models (NIF experiments). We note that two of these three EOS models are Perdew-BurkeErnzerhof (PBE)-based global models. It is well known that PBE [9] systematically underestimates the pressure conditions necessary for dissociation and thus will predict isentropes that exhibit regions of strong $-d T / d P$ (due to the latent heat of the transition) at pressures well below the actual metallization boundary; any global model that builds in latent heat well before the transition will underestimate the experimental 
temperature, perhaps by as much as several hundred kelvins. With this caveat, the resulting estimates of the temperature on encountering the phase boundary were comparable for both sets of experiments, thus presenting an $\sim 95-\mathrm{GPa}$ difference in the location of the metallization boundary at comparable temperatures.

In an effort to reconcile this significant difference in metallization pressure, Celliers et al. argue the NIF experiments probe the entrance of a given isentrope to the coexistence region, while the $\mathrm{Z}$ experiments probe the exit from the coexistence region. This interpretation, as it stands, is consistent with the difference in the indicators used for identification of the phase boundary in the two sets of experiments: The rise of reflectivity with increasing pressure in the NIF experiments and the drop of reflectivity on the descent from high pressure in the $\mathrm{Z}$ experiments. However, it is further argued in Ref. [8] that a factor of 2 downward correction to the inferred temperatures reported by Knudson et al. [7] is required due to a very large latent heat effect. This reanalysis, formally based on the Clausius-Clapeyron relation, but equivalent to requiring that the two well-separated pressure points $(\Delta P \sim 95 \mathrm{GPa})$ lie on the same phase boundary, would require that the temperature estimates in the $\mathrm{Z}$ experiments decrease by $\sim 600 \mathrm{~K}$ to nearly $900 \mathrm{~K}$ for the lowest- and highest-temperature loading paths, respectively. Subject to this reinterpretation, both sets of experimental data would be in quite good agreement with recent coupled electron-ion Monte Carlo (CEIMC) calculations for the phase boundary reported by Pierleoni et al. [10] and prior density functional calculations with the original van der Waals density functional (vdW-DF1) [7]. While this result would be quite appealing from a theoretical point of view, we will show here that under more careful consideration this reinterpretation is in marked disagreement with the thermodynamics of the transition within a first-principles framework.

In particular, the Clausius-Clapeyron analysis presented in Ref. [8], by itself, is incomplete, as it does not include any constraints imposed by the thermodynamics of the phase transition. For a given phase boundary the pressure difference for the entrance and exit of an isentrope, the effective specific heat along the phase boundary, and the latent heat are not arbitrary, but rather dictated by thermodynamics. Here we demonstrate that while the various first-principles frameworks disagree on the location of the first-order transition boundary in $(P, T)$ space, they are in quite good agreement on the thermodynamics of the transition (i.e., the specific heats, Grüneisen gamma, bulk modulus, etc.). Furthermore, we show that subject to the thermodynamics of the transition, the large temperature drop suggested by Celliers et al. [8] is not thermodynamically consistent; such a large temperature drop would require an anomalously low specific heat for the metallic hydrogen phase. An initial critique of these temperature corrections on thermodynamic grounds was presented in Desjarlais et al. [11]; counterarguments were presented in Celliers et al. [12]. Here we provide a more detailed analysis, including nuclear quantum effects.

\section{PHASE BOUNDARY THERMODYNAMICS}

To systematically address the question of the temperature drop resulting from the latent heat of the insulator-metal

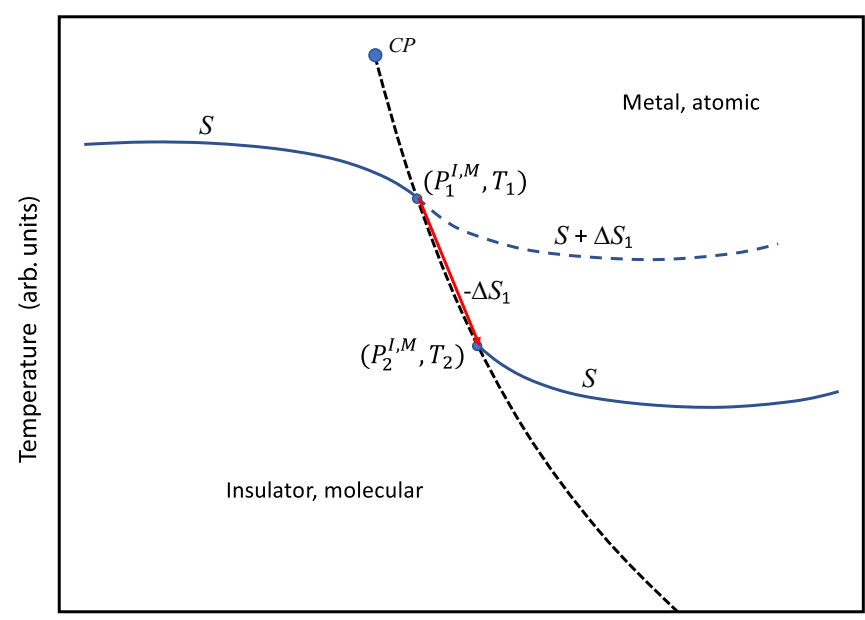

Pressure (arb. units)

FIG. 1. Notional schematic of the PT phase diagram for deuterium around the insulator-metal transition with representative isentropes. The black dashed line represents the phase boundary and terminates at the critical point. The isentropes are depicted with slight $-d T / d P$ near the phase boundary, resulting from a negative Grüneisen $\gamma$, in accordance with first-principles calculations. The red arrow suggests a convenient integration path from $S+\Delta S_{1}$ back to $S$.

transition, we start with the assumption, common to both papers cited above, that the experimental path follows an isentrope. Figure 1 is a schematic for the phase boundary and representative isentropes in $(P, T)$ space. As shown in Fig. 1, the isentropes exhibit a negative slope close to the transition, consistent with a negative Grüneisen $\gamma$. See, for example, the isentropes obtained by thermodynamic integration in Fig. 1 of Knudson et al. [7]. For an isentrope $S$ that enters the coexistence region at a point $\left(P_{1}^{I}, T_{1}\right)$ and a given latent heat $\Delta H_{1}=T_{1} \Delta S_{1}$ we can readily compute the entropy at $\left(P_{1}^{M}, T_{1}\right)$ as $S+\Delta H_{1} / T_{1}=S+\Delta S_{1}$. Here the superscripts $I$ and $M$ refer to points on the insulator and metallic sides of the coexistence region, respectively. To obtain the temperature $T_{2}$ at which the isentrope $S$ exits the coexistence region at $\left(P_{2}^{M}, T_{2}\right)$ we need only compute the temperature drop required to remove the excess entropy $\Delta S_{1}$ in going from $\left(P_{1}^{M}, T_{1}\right)$ to $\left(P_{2}^{M}, T_{2}\right)$. Note that this path is a convenient integration path and should not be confused with the actual experimental thermodynamic path, as was done in Celliers et al. [12].

We derive in the following the exact expression for change in entropy along a line in $(P, T)$ space in terms of quantities readily calculated within an $N V T$ first-principles framework. We start with the total derivative of $S(P, T)$ :

$$
d S=\left(\frac{\partial S}{\partial T}\right)_{P} d T+\left(\frac{\partial S}{\partial P}\right)_{T} d P .
$$

We can specify an arbitrary line in $(P, T)$ space by imposing the linear constraint

$$
d P=\left(\frac{d P}{d T}\right)_{\text {line }} d T
$$

This results in

$$
\left(\frac{d S}{d T}\right)_{\text {line }}=\frac{C_{P}}{T}+\left(\frac{\partial S}{\partial P}\right)_{T}\left(\frac{d P}{d T}\right)_{\text {line }},
$$


where we have used the definition

$$
\left(\frac{\partial S}{\partial T}\right)_{P}=\frac{C_{P}}{T}
$$

where $C_{P}$ is the specific heat at constant pressure. We can further reduce this in terms of quantities that are readily calculable with the Maxwell relation

$$
\left(\frac{\partial S}{\partial P}\right)_{T}=-\left(\frac{\partial V}{\partial T}\right)_{P}=\left(\frac{\partial P}{\partial T}\right)_{V} /\left(\frac{\partial P}{\partial V}\right)_{T} .
$$

Given the definitions

$$
\gamma=\frac{V}{C_{V}}\left(\frac{\partial P}{\partial T}\right)_{V}=V\left(\frac{\partial P}{\partial E}\right)_{V}, \quad \frac{B_{T}}{V}=-\left(\frac{\partial P}{\partial V}\right)_{T},
$$

where $B_{T}$ is the isothermal bulk modulus, $C_{V}$ is the specific heat at constant volume, and $\gamma$ is the Grüneisen $\gamma$, we arrive at

$$
\left(\frac{d S}{d T}\right)_{\text {line }}=\frac{C_{P}}{T}\left[1-\frac{C_{V}}{C_{P}} \frac{\gamma T}{B_{T}}\left(\frac{d P}{d T}\right)_{\text {line }}\right] .
$$

With calculations of $C_{V}, \gamma$, and $B_{T}, C_{P}$ is readily obtained via $C_{P}=C_{V}\left(1+\gamma^{2} T C_{V} / V B_{T}\right)$.

In Celliers et al. [12] expression (7) was noted to be equivalent to Eq. (4.19) in Reichl [13], but with the erroneous interpretation that this expression is to be applied only at constant volume. As is clear from the derivation, this expression for the change in entropy is completely general for a given line in $(P, T)$ space. For the specific application here, this expression is applied along a line adjacent to, but on the metallic side of, the phase transition, as indicated by the red arrow in Fig. 1. For the slope of the coexistence boundary in the vicinity of 1300 to $1400 \mathrm{~K}$, we use $d P / d T_{\text {coex }}=-0.12$ $\mathrm{GPa} / \mathrm{K}$ as an average, which agrees well with the published coexistence line for vdW-DF1, including nuclear quantum effects, around that temperature range [7]. $C_{V}, \gamma$, and $B_{T}$ are determined from first-principles calculations.

\section{FIRST-PRINCIPLES CALCULATIONS WITH NUCLEAR QUANTUM EFFECTS}

To obtain quantitative values for $C_{V}, \gamma$, and $B_{T}$, including nuclear quantum effects, we have performed several pathintegral molecular dynamics (PIMD) calculations with finitetemperature density functional theory (DFT) as implemented in VASP 5.3.5 [14-16], using the PIMD scheme of Alfè and Gillan [17]. This implementation of PIMD is based on the well-known isomorphism [18] to a ring polymer chain wherein individual imaginary time slices or images are represented by $\mathrm{P}$ beads on a polymer chain coupled by harmonic springs. The path integral is discretized with the primitive Trotter expansion (second order in the imaginary time step $\tau=\hbar / k_{B} T \mathrm{P}$ ). To overcome the inefficient (nonergodic [19]) thermal sampling of the ring polymer harmonic vibrational modes when all beads on the chain are given the same mass, a mass matrix technique is used to collapse the widely spread normal modes of the ring to a narrow frequency spectrum and accelerate energy exchange between modes. We note that this implementation of PIMD is generally applicable to the computation of stationary properties of the quantum canonical ensemble, such as total energy, pressure, and structure factors, but not dynamical properties such as diffusion coefficients and other transport properties. In practice, $\mathrm{P}$ simulations are launched in parallel, with each PIMD image running on multiple processors, typically 36 cores per image in the calculations presented here.

The DFT-PIMD calculations were performed with the vdW-DF1 [20] exchange-correlation functional. Note the choice of vdW-DF1 for these calculations is motivated by arguments in Celliers et al. [8] that (i) the onset of the phase transition is in close agreement with the phase boundary predicted by either CEIMC or DFT calculations with vdW-DF1 and nuclear quantum effects and (ii) the $\mathrm{Z}$ experiments are indicative of the exit from that boundary. Therefore vdW-DF1 is a logical choice for directly addressing the thermodynamics of their argument in that region of phase space in a density functional framework. However, as discussed in Knudson et al. [7], the phase boundary suggested by the Z experiments is in better agreement with the vdW-DF2 functional, and the effect of the latent heat was implicitly computed in Knudson et al. [7] through direct calculation of the isentropes on both sides of the phase boundary by thermodynamic integration.

Each DFT-PIMD calculation consists of $\mathrm{P}=8$ pathintegral molecular dynamics images (beads) for a Trotter time step $\leqslant 9.5 \times 10^{-5} \mathrm{~K}^{-1}$. Morales et al. [21] found convergence with eight images for protons at $1000 \mathrm{~K}$ within the primitive Trotter discretization. Convergence here is further aided by the higher deuterium mass and higher temperatures. More quantitatively, we consider a well-known criterion for energy or pressure convergence of PIMD calculations in the primitive Trotter breakup (see, for example, Ref. [22]); the number of images $\mathrm{P}$ should be at least some small multiple of $h v_{\max } / k_{B} T$, where $v_{\max }$ is a significant high frequency in the system, in this case the $\mathrm{D}_{2}$ vibron frequency. For our calculations $\mathrm{P} /\left(h \nu_{\max } / k_{B} T\right) \sim 2.7$, versus $\sim 1.5$ for the Morales calculations. Further aiding the convergence for both of these systems is the location of the bulk of the modes of the system well below the vibron frequency. A few test calculations were performed with 16 and 32 images and confirmed the convergence of the energy and pressure with 8 images.

Each PIMD image contains 256 deuterium atoms represented with a projector augmented-wave potential [23,24]. The Brillouin zone was sampled at the Baldereschi mean value point [25], and the plane wave cutoff energy was $700 \mathrm{eV}$. Individual DFT-PIMD runs consisted of 4000 time steps of $0.25 \mathrm{fs}$, for a total simulated time of $1 \mathrm{ps}$. An Andersen thermostat [26] was employed to regulate the temperature and approximate a canonical ensemble. Due to the relatively short nature of each individual run, we observed statistical variation in the temperature about the target temperature, along with expected statistical variation in the thermodynamic quantities. To minimize the statistical variance in the thermodynamic averages, generalized virial estimators [27] were used for the pressure and energy.

The results of these calculations for specific volumes of $1.80 \AA^{3} /$ atom (green circles, dotted fits) and $1.78 \AA^{3} /$ atom (black circles, solid fits) are illustrated in Fig. 2 as the total energy versus temperature and in Fig. 3 as pressure times volume versus the energy, providing $C_{V}$ and $\gamma$, respectively. The lower temperature bound of the data set for each fit was chosen to correspond to a sharp drop in the dimer peak of the 


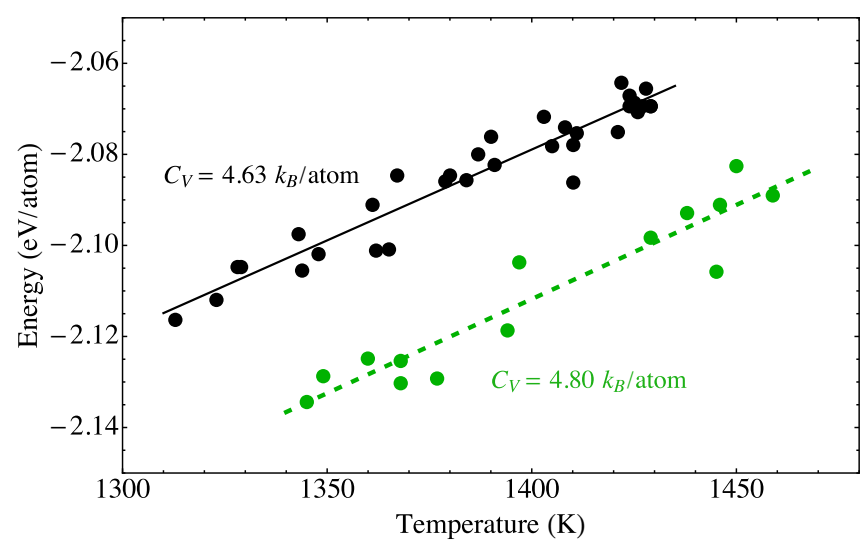

FIG. 2. Energy versus temperature in the vicinity of the insulator-metal transition at specific volumes of $1.80 \AA^{3} /$ atom (green circles, dotted fit) and $1.78 \AA^{3}$ /atom (black circles, solid fit). $C_{V}$ is given by the slope.

pair correlation function, as computed from the path centroid positions and indicative of the molecular to atomic transition. By this construction we are performing calculations adjacent to the metallic side of the phase transition in the region traversed by the path from $\left(P_{1}^{M}, T_{1}\right)$ to $\left(P_{2}^{M}, T_{2}\right)$ suggested by the red arrow in Fig. 1. For a specific volume of $1.80 \AA^{3} /$ atom we find a transition at $\sim 1345 \mathrm{~K}$ and $P=204 \mathrm{GPa}$. For a specific volume of $1.78 \AA^{3}$ /atom we find a transition at $\sim 1310 \mathrm{~K}$ and $P=208.5 \mathrm{GPa}$. These $(P, T)$ phase transition points are in excellent agreement with the CEIMC predictions in Pierleoni et al. [10] for $\mathrm{D}_{2}$ with quantum deuterons as well as the estimate from Knudson et al. [7] for vdW-DF1 based on equating the spatial width of the classical and quantum $\mathrm{D}_{2}$ dimer peaks. From the linear fits to the DFT-PIMD data we extract values of $C_{V}=4.80 k_{B} /$ atom and $\gamma=-1.21$ for $V=1.80 \AA^{3} /$ atom and $C_{V}=4.63 k_{B} /$ atom and $\gamma=-1.16$ for $V=1.78 \AA^{3} /$ atom.

To determine $B_{T}$ adjacent to the phase boundary, we have computed $P$ versus $T$ over a wide range of volumes and temperatures, as shown in Fig. 4. From the individual fits to

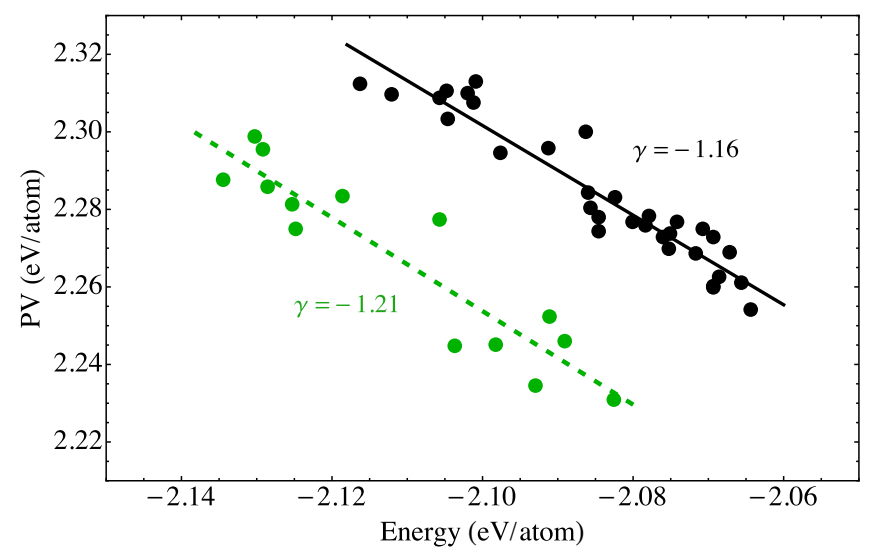

FIG. 3. Pressure times volume versus energy in the vicinity of the insulator-metal transition at specific volumes of $1.80 \AA^{3} /$ atom (green circles, dotted fit) and $1.78 \AA^{3} /$ atom (black circles, solid fit). The Grüneisen $\gamma$ is given by the slope.

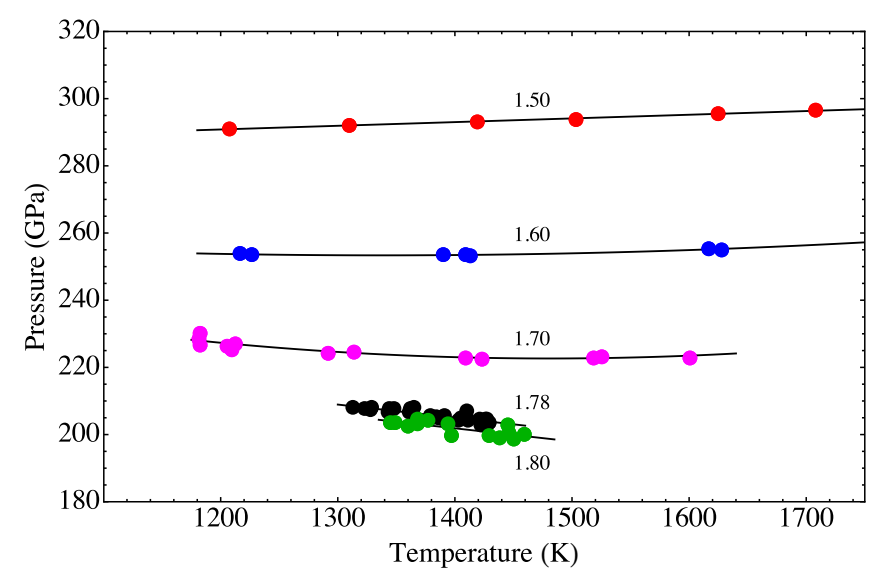

FIG. 4. Pressure versus temperature for several specific volumes. Labels for each fit indicate the specific volume (in $\AA^{3} /$ atom).

the pressure, we generate $P$ versus $V$ at $1345 \mathrm{~K}$, as shown by the corresponding second-order polynomial fit in Fig. 5. Differencing the pressure fit at $V=1.80 \AA^{3} /$ atom and using the second definition in Eq. (6) yields $B_{T}=272 \mathrm{GPa}$. We find that $B_{T}$ increases rapidly over the course of several tens of $\mathrm{GPa}$, reaching $540 \mathrm{GPa}$ within $50 \mathrm{GPa}$ at $1345 \mathrm{~K}$; however, it is this lower local value that is relevant to the calculation along the phase boundary and which slightly depresses the effective specific heat along the coexistence line.

From $C_{P}=C_{V}\left(1+\gamma^{2} T C_{V} / V B_{T}\right)$, with $T=1345 \mathrm{~K}$ and $V=1.80 \AA^{3} /$ atom, we find $C_{P}=6.07 k_{B}$ /atom. Combining terms and inserting in Eq. (7), we find

$$
\left(\frac{d S}{d T}\right)_{\mathrm{coex}}=\frac{C_{P}}{T}\left[1-\frac{C_{V}}{C_{P}} \frac{\gamma T}{B_{T}}\left(\frac{d P}{d T}\right)_{\mathrm{coex}}\right] \equiv \frac{C_{\mathrm{coex}}}{T},
$$

with $C_{\text {coex }}=2.64 k_{B}$ /atom. Integrating Eq. (8) with the constraint $\int_{1}^{2} d S=-\Delta S_{1}$ and assuming $C_{\text {coex }}$ is weakly varying or represents an average value give

$$
\frac{T_{2}}{T_{1}} \cong \exp \left(-\Delta S_{1} / C_{\mathrm{coex}}\right)
$$

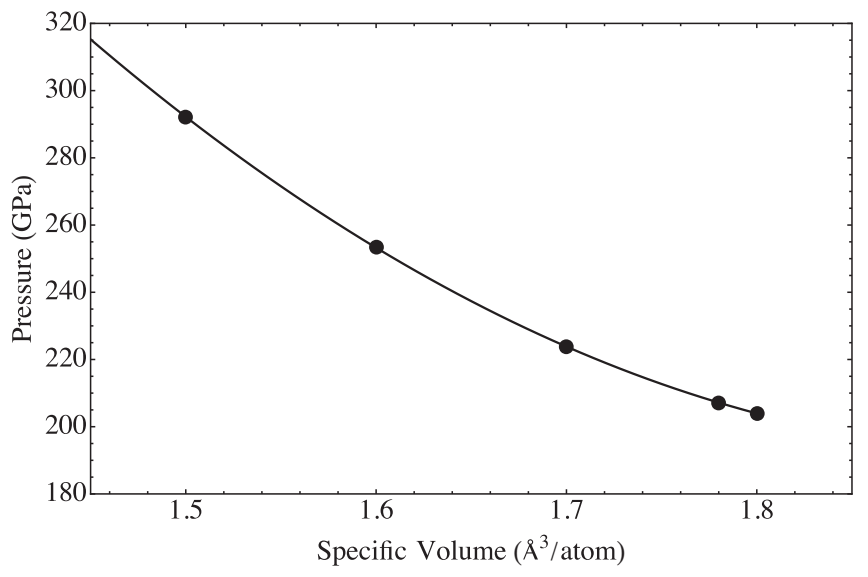

FIG. 5. Pressure versus specific volume at $1345 \mathrm{~K}$. 


\section{ISENTROPIC TEMPERATURE DROP DUE TO LATENT HEAT}

There are previous calculations of the latent heat for this insulator-metal transition in the literature [10,21]. We obtain estimates of the latent heat by searching for pairs of calculations at the same pressure (within 0.5\%) and temperature (within 1\%), but distinctly in the molecular and atomic phases at the phase boundary. These pairs were successfully obtained with a $2.2 \%$ density difference between the molecular and atomic phases, consistent with the findings of Refs. [10,21] for the width of the coexistence region. Differencing the enthalpy of these phase boundary pairs gives results consistent with those of Pierleoni et al. [10] for quantum protons: A latent heat of $\sim 0.05 \pm 0.005 \mathrm{eV} /$ atom (or 522 to $638 \mathrm{~K}$ ) over the temperature range of $1300-1350 \mathrm{~K}$. Using $\Delta H_{1}=$ $0.05 \mathrm{eV} /$ atom for the latent heat and $T_{1}=1345 \mathrm{~K}$ sets $\Delta S_{1}=$ $0.431 k_{B}$ /atom. For $C_{\text {coex }}=2.64 k_{B} /$ atom, as calculated above at $V=1.80 \AA^{3} /$ atom, $T_{2} / T_{1}=0.85$, according to Eq. (9). We do not find these calculations very sensitive to the $0.050 \pm$ $0.005 \mathrm{eV} /$ atom extrema quoted for the latent heats, producing only a $\mp 1.5 \%$ change in $T_{2} / T_{1}$. These results are similarly insensitive to the slope of the phase boundary, with a $\pm 10 \%$ change in the magnitude of $d P / d T_{\text {coex }}$ producing a $\mp 2 \%$ change in $T_{2} / T_{1}$.

These final results are only very weakly dependent on the explicit $T$ dependence within the square brackets of Eq. (8), so for the purposes of computations above we have treated $T$ as a constant equal to $T_{1}$. Because of the weak dependence, iteratively replacing $T$ by $\bar{T}=\left(T_{1}+T_{2}\right) / 2$ converges very rapidly. As a specific quantitative example of this, consider a case where the calculated $C_{\text {coex }}=2.64 k_{B} /$ atom at $V=$ $1.80 \AA^{3}$ /atom is assumed to represent a midpoint or average value $\bar{C}_{\text {coex }}$ within the temperatures spanned from $T_{1}$ to $T_{2}$, where $T_{2} / T_{1}=\exp \left(-\Delta S_{1} / \bar{C}_{\text {coex }}\right)$. Iterating the above equations as described, with the target $\bar{T}=\left(T_{1}+T_{2}\right) / 2=1345 \mathrm{~K}$, results in a solution with $T_{1}=1447 \mathrm{~K}, \Delta S_{1}=0.401 k_{B} /$ atom, $T_{2}=1243 \mathrm{~K}$, and $T_{2} / T_{1}=0.86$. For a phase boundary slope of $d P / d T_{\text {coex }}=-0.12 \mathrm{GPa} / \mathrm{K}$, this calculated $\Delta T=202 \mathrm{~K}$ along the coexistence boundary would correspond to $\Delta P=$ $24 \mathrm{GPa}$. Comparing these results to the green curve in Fig. 1 of Knudson et al. [7] reveals very good agreement with the latent-heat-induced temperature drop obtained there by thermodynamic integration using the vdW-dF2 functional [28]. Furthermore, Fig. 4c (green curve) of Knudson et al. [7], which shows reflectivity as a function of pressure and corresponds to these same approximate conditions, is in good agreement with this $\Delta P$ estimate on the fast rising linear portion of the reflectivity history.

It is interesting to note that despite the relatively high value of $C_{P}=6.07 k_{B} /$ atom found on the metallic side of the phase boundary, the negative slope of the phase boundary and the negative slope of the isentrope near the boundary $(\gamma<0)$ combine to give an effective specific heat through Eq. (9) that is very close to what we find well away from the phase boundary in the region where $\gamma \sim 0$. In that region, at a specific volume of $1.60 \AA^{3}$ /atom and $1345 \mathrm{~K}$ (around $254 \mathrm{GPa}$ in Fig. 4), the specific heat $C_{P} \approx C_{V}=2.61 k_{B} /$ atom. As was noted in Desjarlais et al. [11], values of specific heat of this magnitude are expected for liquid alkali metals; $C_{P}=3.5 k_{B}$ /atom for liquid lithium metal [29].

\section{DISCUSSION}

Given the close agreement between (i) the results presented here using vdW-DF1, (ii) the estimates provided in Desjarlais et al. [11] obtained using interpolations on CEIMC data from Pierleoni et al. [10], and (iii) the direct isentrope calculations presented in Knudson et al. [7] obtained through thermodynamic integration using vDW-DF2, we wish to comment now on the apparent discrepancy between these results and the estimated temperature reduction factor $T_{2} / T_{1}=0.66$ presented by Celliers et al. [12]. Their estimate was obtained from analysis of their Eqs. (1) and (2). However, in computing their Eq. (2), $\gamma$ was incorrectly treated as a constant over the entire $95 \mathrm{GPa}$ range of the integral. As is clear from the temperature minimum of the isentropes obtained by thermodynamic integration in Fig. 1 of Knudson et al. [7] and suggested also by Fig. S32 of the Supplemental Material for Celliers et al. [8] along with Fig. 1 of Celliers et al. [12], the Grüneisen $\gamma \rightarrow 0$ and eventually turns positive over the course of several tens of gigapascals beyond the phase boundary. This follows directly from $(\partial S / \partial P)_{T}=-\gamma C_{V} / B_{T}$ and is a direct consequence of exhausting the molecular to atomic transition. That $\gamma \rightarrow 0$ within approximately $50 \mathrm{GPa}$ is illustrated in Fig. 4, where $(\partial P / \partial T)_{V}$ is essentially zero by $255 \mathrm{GPa}$ for temperatures between 1200 and $1400 \mathrm{~K}$. Correcting the integration in Eq. (2) of Ref. [12] and accounting for the consequences in subsequent steps in that analysis bring those estimates in line with the results obtained here.

A refinement of the finite-difference analysis outlined in Ref. [8] is presented in Ref. [12]. This analysis combines the Clausius-Clapeyron relation with the assumption that the $\mathrm{NIF}$ and $\mathrm{Z}$ pressures are indicative of the entrance $\left(P_{1}^{I}, T_{1}\right)$ and exit $\left(P_{2}^{M}, T_{2}\right)$ of the coexistence region. By effectively constraining the 95-GPa pressure difference to the coexistence line, they arrive at a temperature reduction factor due to latent heat of $T_{2} / T_{1}=0.58$. This value of 0.58 , when combined with Eq. (9), and $T_{1}=1447 \mathrm{~K}$ imply an anomalously low $C_{\text {coex }}=0.74 k_{B} /$ atom, in gross disagreement with the $C_{\text {coex }}=$ $2.64 k_{B} /$ atom obtained from our first-principles calculations. We emphasize that this larger value for $C_{\text {coex }}$ is consistent across vdW-DF1, vdW-DF2, and CEIMC; even though these different first-principles frameworks disagree on the precise location of the insulator-metal phase transition boundary, they are in quite good agreement on the thermodynamics of the transition (i.e., the specific heats, Grüneisen $\gamma$, bulk modulus, etc.).

As noted in the Introduction, the criteria for identifying the location of the phase boundary are substantively different between the $\mathrm{Z}$ and NIF experiments. In the $\mathrm{Z}$ experiments the phase boundary was associated with the abrupt drop from a high-reflectivity phase upon pressure release. The NIF experiments probed only the transition upon compression; there the phase boundary was associated with the reflectivity rising above a threshold of $30 \%$. Both approaches are valid; however, comparing one to the other on unequal footing exacerbates the apparent discrepancy. Associating the phase boundary in the NIF experiments with a higher value of reflectivity, commensurate with completion of the transition, would result in a higher inferred value for the transition pressure. As a quantitative illustration, consider the calculations of Rillo 
et al. [30], which suggest a reflectivity in excess of $40 \%$ at the completion of the transition at a temperature of $1500 \mathrm{~K}$. Applying these criteria to the $1450 \mathrm{~K}$ (closest available and, specifically, N150914-2) reflectivity versus pressure trace in Fig. 2 of Celliers et al. [8] suggests a phase boundary exit pressure of $240 \mathrm{GPa}$.

Evaluating the completion of the transition would also result in a lower inferred temperature, in accordance with the latent heat considerations explored here. However, regarding the estimated temperature, as noted in the Introduction, two of the three equation of state models used to infer the temperature in the NIF experiments include PBE latent heat contributions at pressures below the observed transition pressure (in either the $\mathrm{Z}$ or NIF interpretations) and therefore result in lower predicted temperatures. The 2003 deuterium EOS of Kerley [31], the same EOS used for estimating temperatures for the $\mathrm{Z}$ experiments prior to switching to DFT calculations of the isentrope, is much closer to, and slightly exceeds, the upper bound of the temperature estimates provided in Fig. 3 of Celliers et al. [8]. Calculation of the temperature path for this reported $1450 \mathrm{~K}$ transition case with the Kerley 2003 EOS is shown in Fig. S18 of the Supplemental Material of Celliers et al. [8]; subtracting $200 \mathrm{~K}$ for latent heat at $240 \mathrm{GPa}$, as indicated by the calculations in this paper, suggests a temperature closer to $1625 \mathrm{~K}$. We note that a transition at $240 \mathrm{GPa}$ and $1625 \mathrm{~K}$ is much closer to the vdW-DF2 phase boundary than that of vdW-DF1. Interpreting this NIF experiment on the same footing as the $\mathrm{Z}$ experiments reduces the apparent discrepancy between the two experiments by about a factor of 2 , to approximately $42 \mathrm{GPa}$. The remaining differences are not insignificant but will require future experiments and analyses to reconcile.

\section{CONCLUSION}

We have performed an extensive study of the thermodynamics of the insulator-metal transition in dense liquid deuterium within a first-principles framework to assist in interpreting recent dynamic compression experiments. Specifically, we used density functional theory, including nuclear quantum effects, to directly calculate the temperature drop for an isentrope that traverses the first-order insulator-metal transition. This was accomplished by evaluating an exact expression for change in entropy along a line in $(P, T)$ space in terms of quantities readily calculated within an $N V T$ first-principles framework. An extensive set of path-integral molecular dynamics calculations with the vdW-DF1 functional was performed to obtain quantitative values for $C_{V}$, $\gamma$, and $B_{T}$ adjacent to the metallic side of the phase transition in the region traversed in recent dynamic compression experiments. The resulting temperature drops were found to be consistent with previous direct isentrope calculations [7] obtained through thermodynamic integration and estimates [11] based on interpolations of CEIMC data [10]. Furthermore, these temperature drops are in stark disagreement with a recent reinterpretation presented by Celliers et al. $[8,12]$

The arguments presented in Celliers et al. $[8,12]$ have their root in constraining both the $\mathrm{Z}$ and NIF experiments to a given theoretical phase boundary and approximate isentrope, with the NIF experiments marking the entrance to the coexistence region and the $\mathrm{Z}$ experiments probing the exit. For the quoted pressures in the two sets of experiments, forcing the $\mathrm{Z}$ experiments to the phase boundary would require a factor of 2 correction downward in the temperature estimates for the $\mathrm{Z}$ experiments. However, as is clear from the analysis presented here, any given pressure difference between the two experiments would, by this enforced temperature constraint, result in a different effective specific heat along the phase boundary, generally in conflict with the underlying thermodynamics. The large temperature corrections required for the estimated 95-GPa pressure difference would necessitate an anomalously low specific heat that is in gross disagreement with firstprinciples calculations presented here using vdW-DF1, those in Pierleoni et al. [10] using CEIMC as argued in Desjarlais et al. [11], and our earlier direct calculations of the isentropes [7] obtained through thermodynamic integration using vdWDF2. While interpreting the $1450 \mathrm{~K}$ NIF experiment in a manner analogous to that used for the $\mathrm{Z}$ experiments suggests a substantially smaller discrepancy between the two sets of experiments, an approximately 42-GPa difference remains. What is clear from the analysis presented here is that the pressure difference cannot be explained through the supposition of a very large latent heat effect between the onset and completion of the phase transition.

\section{ACKNOWLEDGMENTS}

M.P.D. would like to thank D. Alfè for providing many of the path-integral algorithms. Sandia National Laboratories is a multimission laboratory managed and operated by National Technology and Engineering Solutions of Sandia, LLC., a wholly owned subsidiary of Honeywell International Inc. for the U.S. Department of Energy's National Nuclear Security Administration under Contract No. DE-NA0003525. This paper describes objective technical results and analysis. Any subjective views or opinions that might be expressed in the paper do not necessarily represent the views of the U.S. Department of Energy or the U.S. Government. R.R. also thanks the Deutsche Forschungsgemeinschaft (DFG) for support via SFB 652 and FOR 2440.
[1] V. Dzyabura, M. Zaghoo, and I. F. Silvera, Proc. Natl. Acad. Sci. USA 110, 8040 (2013).

[2] K. Otha, K. Ichimaru, M. Einaga, S. Kawaguchi, K. Shimizu, T. Matsuoka, N. Hirao, and Y. Ohishi, Sci. Rep. 5, 16560 (2015).

[3] M. Zaghoo, A. Salamat, and I. F. Silvera, Phys. Rev. B 93, 155128 (2016).
[4] R. S. McWilliams, D. A. Dalton, M. F. Mahmood, and A. F. Goncharov, Phys. Rev. Lett. 116, 255501 (2016).

[5] M. Zaghoo and I. F. Silvera, Proc. Natl. Acad. Sci. USA 114, 11873 (2017).

[6] R. P. Dias and I. F. Silvera, Science 355, 715 (2017). 
[7] M. D. Knudson, M. P. Desjarlais, A. Becker, R. W. Lemke, K. R. Cochrane, M. E. Savage, D. E. Bliss, T. R. Mattsson, and R. Redmer, Science 348, 1455 (2015).

[8] P. M. Celliers, M. Millot, S. Brygoo, R. S. McWilliams, D. E. Fratanduono, J. R. Rygg, A. F. Goncharov, P. Loubeyre, J. H. Eggert, J. L. Peterson, N. B. Meezan, S. L. Pape, G. W. Collins, R. Jeanloz, and R. J. Hemley, Science 361, 677 (2018).

[9] J. P. Perdew, K. Burke, and M. Ernzerhof, Phys. Rev. Lett. 77, 3865 (1996).

[10] C. Pierleoni, M. A. Morales, G. Rillo, M. Holzmann, and D. M. Ceperley, Proc. Natl. Acad. Sci. USA 113, 4953 (2016).

[11] M. P. Desjarlais, M. D. Knudson, and R. Redmer, Science 363, eaaw0969 (2019).

[12] P. M. Celliers, M. Millot, S. Brygoo, R. S. McWilliams, D. E. Fratanduono, J. R. Rygg, A. F. Goncharov, P. Loubeyre, J. H. Eggert, J. L. Peterson, N. B. Meezan, S. L. Pape, G. W. Collins, R. Jeanloz, and R. J. Hemley, Science 363, eaaw1970 (2019).

[13] L. E. Reichl, A Modern Course in Statistical Physics (University of Texas, Austin, 1980).

[14] G. Kresse and J. Hafner, Phys. Rev. B 47, 558 (1993).

[15] G. Kresse and J. Hafner, Phys. Rev. B 49, 14251 (1994).

[16] G. Kresse and J. Furthmüller, Phys. Rev. B 54, 11169 (1996).

[17] D. Alfè and M. J. Gillan, J. Chem. Phys. 133, 044103 (2010).
[18] D. Chandler and P. G. Wolynes, J. Chem. Phys. 74, 4078 (1981).

[19] W. Hall and B. J. Berne, J. Chem. Phys. 81, 3641 (1984).

[20] M. Dion, H. Rydberg, E. Schröder, D. C. Langreth, and B. I. Lundqvist, Phys. Rev. Lett. 92, 246401 (2004).

[21] M. A. Morales, C. Pierleoni, E. Schwegler, and D. M. Ceperley, Proc. Natl. Acad. Sci. USA 107, 12799 (2010).

[22] T. E. Markland and M. Ceriotti, Nat. Rev. Chem. 2, 0109 (2018).

[23] P. E. Blöchl, Phys. Rev. B 50, 17953 (1994).

[24] G. Kresse and D. Joubert, Phys. Rev. B 59, 1758 (1999).

[25] A. Baldereschi, Phys. Rev. B 7, 5212 (1973).

[26] H. C. Andersen, J. Chem. Phys. 72, 2384 (1980).

[27] M. Parrinello and A. Rahman, J. Chem. Phys. 80, 860 (1984).

[28] K. Lee, E. D. Murray, L. Kong, B. I. Lundqvist, and D. C. Langreth, Phys. Rev. B 82, 081101(R) (2010).

[29] M. W. Chase Jr. (Ed.), NIST-JANAF Thermochemical Tables, Fourth Edition (American Institute of Physics, Woodbury, New York, 1998).

[30] G. Rillo, M. A. Morales, D. M. Ceperley, and C. Pierleoni, Proc. Natl. Acad. Sci. USA 116, 9770 (2019).

[31] G. Kerley, Sandia National Laboratories, Report No. SAND2003-3613, 2003 (unpublished). 